\title{
Vector-borne diseases and the basic reproduction number: a case study of African horse sickness
}

\author{
C. C. LORD, M. E. J. WOOLhOUSE, J. A. P. HEESTERBEEK* and \\ P. S. MELLOR $†$ Department of Zoology, University of Oxford, U.K., *Agricultural Mathematics Group, \\ GLW-DLO, Wageningen, The Netherlands, and †Institute for Animal Health, Pirbright Laboratory, U.K.
}

\begin{abstract}
The basic reproduction number, $R_{0}$, can be used to determine factors important in the ability of a disease to invade or persist. We show how this number can be derived or estimated for vector-borne diseases with different complicating factors. African horse sickness is a viral disease transmitted mainly by the midge Culicoides imicola. We use this as an example of such a vector-transmitted disease where latent periods, seasonality in vector populations, and multiple host types may be important. The effect of vector population dynamics which are dependent on either host or vector density are also addressed. If density-dependent constraints on vector population density are less severe, $R_{0}$ is more sensitive to vector mortality and the virus development rate. Host-dependent vector dynamics change the relationship between $R_{0}$ and host population size. Seasonality can either increase or decrease the estimate of $R_{0}$, depending on the lag between the peak of the midge population and the infective host population. The relative abundance of two host types is a factor in the ability of a disease to invade, but the strength of this factor depends on the differences between the hosts in recovery from infection, mortality and transmission. Removal of a reservoir host may increase $R_{0}$.
\end{abstract}

Key words. Basic reproduction number, mathematical models, African horse sickness, Culicoides imicola.

\section{Introduction}

Vector-borne diseases present a special problem when considering the ability of a disease agent to invade or persist in a new area. The biology of the host, disease agent and vector must be considered, as must the possibility that another species of vector or host will be able to transmit the disease. It is often difficult to determine or predict what factors will be the most important in the invasion or persistence of a disease agent.

There have been many theoretical studies of vector-borne disease, starting with the Ross-Macdonald malaria model (Ross, 1911; Macdonald, 1957; Anderson \& May, 1991, and references therein). One use of these models is to explore which parameters are most important for disease invasion and the behaviour of epidemics following invasion. One concept developed from theoretical studies is the basic reproduction number, $R_{0}$, also referred to as the 'basic reproductive rate' (Anderson \& May, 1991) or the 'basic reproduction ratio' (Diekmann et al., 1990; Scott \&

Correspondence: Dr C. Lord, Department of Zoology, South Parks Road, Oxford OX1 3PS, U.K.
Smith, 1994). $R_{0}$ is defined as the average number of infected hosts resulting from the introduction of one infected host into a susceptible population. From this definition, it is clear that if $R$ is less than 1 the disease cannot invade or persist in the host population. Mathematical expressions for $R_{0}$ can be used as a guide to empirical research, focusing study on factors which are most likely to affect the potential for invasion. $R_{0}$ also provides a guide to the intensity of control efforts needed to prevent disease epidemics, such as the fractional reduction in vector population density or the proportion of hosts which must be vaccinated (Anderson \& May, 1991).

African horse sickness (AHS) is an example of a disease where there are multiple complicating factors. The causative virus (Reoviridae: Orbivirus) can infect most species of equines, so we are concerned with at least two species of host (horses and donkeys), and potentially a third as well (zebra). The most common vector, the biting midge Culicoides imicola Kieffer (Diptera: Ceratopogonidae), also feeds on mammals that are not susceptible to the virus. The vector has seasonal fluctuations in its abundance, the presumed cause of seasonal variation in disease prevalence. The virus is endemic in sub-Saharan Africa, 
but has caused severe epidemics when introduced to naive equine populations outside this area. Identifying factors which affect the ability of the virus to invade would be helpful in predicting areas at risk and in the design of control methods.

We use this disease as an example to show how $R_{0}$ is formulated for a nonseasonal, one- and two-host system and how it can be estimated for a one-host system with seasonality. Using these equations, we explore which parameters are crucial to our understanding of the epidemiology of this disease, which will lead us to recommendations for field and laboratory studies.

\section{Methods and Results}

Basic model

We start with a basic model, of a type that has been used to study several vector-borne diseases (Anderson \& May, 1991). This includes one host species and one vector species, and does not include seasonal variation. As is common for models of viral diseases, this is a compartmental model, with hosts and vectors classified according to whether they are susceptible (i.e. uninfected and not immune), latent (have been infected but are not yet infective), infective, or immune.

For AHS, we can make a number of assumption which simplify the structure of the model (a schematic of the model for AHS is shown in Fig. 1, and a list of parameters in Table 1). As the average latent period in hosts tends to be short compared with the duration of infectiveness and the average host life expectancy, we assume that infected hosts are immediately infective, and so there is no latent class for hosts. As hosts are long-lived relative to all other time periods in the model (such as vector lifespan and duration of infection), we ignore natural host mortality and reproduction. We are then concerned with the proportion of the host population that is in a particular class, rather than the number.

For vectors, however, the latent period and the lifespan may be similar in magnitude. Some vectors may not survive the latent period, and so latency must be included. We again assume a constant population size, but the lifespan of a midge is short relative to other time periods in the model. Therefore we include both reproduction and mortality in the equations describing the midge population. This results in a turnover of the midge population, although the total population size is constant. Vectors are usually infected for life, so we do not need an immune class. We only consider female vectors, as males do not feed on blood. For convenience, we describe the vector population in terms of the number of individuals in each class, rather than the proportion. In the discussion that follows, the number of individuals will be referred to by a capital letter, and the proportion will be referred to with a lowercase letter (e.g. $x$ is the proportion of hosts susceptible and $X$ the number. $X=x^{*} H$, where $H$ is the total number of hosts.)

Susceptible hosts, $x$. Transmission between vectors and hosts depends on the number of bites by a midge on AHS hosts per day, represented by $a$ (with only one host type $1 / a=\alpha$, the average interval (in days) between blood meals on an AHS host); the ratio of midges to hosts, $N / H$ (this gives $a(N / H)$, the number of bites per host per day); the proportion of those midges which are infected, $v$; the proportion of bites by an infected midge that results in an infected host, $b$; and the proportion of hosts which are susceptible, $x$. Therefore,

$$
\frac{d x}{d t}=-a b\left(\frac{N}{H}\right) x v .
$$

\section{HOSTS}

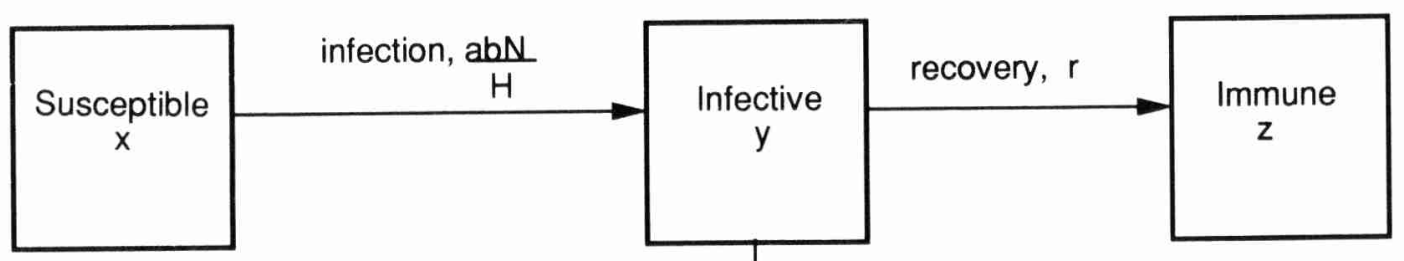

mortality, $c$

\section{VECTORS}

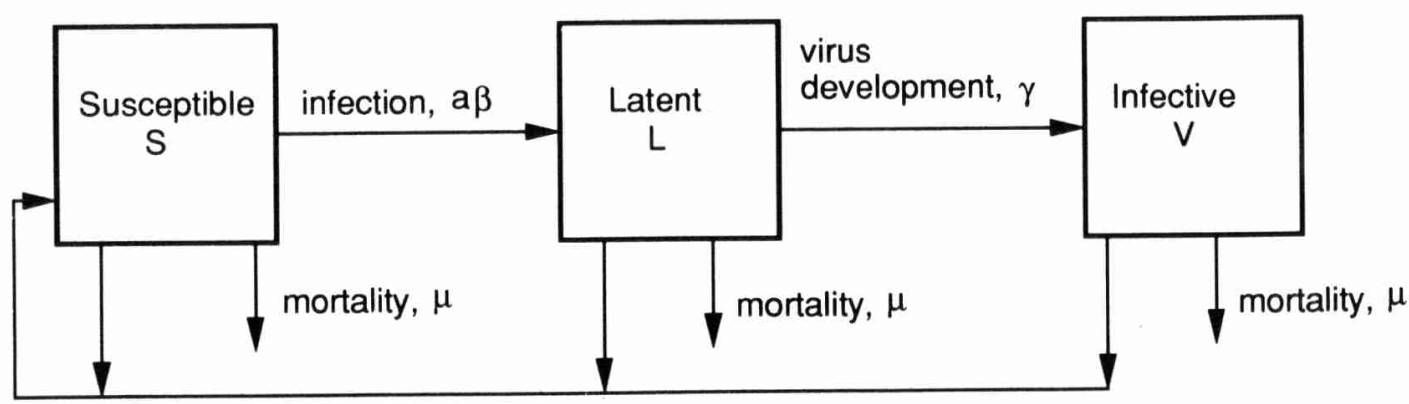

recruitment, $\rho$

Fig. 1. Schematic for the basic one-host model. 
Table 1. Variables and parameters used in models.

\begin{tabular}{|c|c|c|}
\hline Population & Symbol & Description \\
\hline \multicolumn{3}{|l|}{ Variables } \\
\hline \multirow[t]{4}{*}{ Hosts } & $H_{\mathrm{i}}$ & Total number of AHS hosts of species $i$ \\
\hline & $x_{i}$ & Proportion of hosts of species $i$ susceptible \\
\hline & $y_{i}$ & Proportion of hosts of species $i$ infected \\
\hline & $z_{i}$ & Proportion of hosts of species $i$ immune due to recovery \\
\hline \multirow[t]{4}{*}{ Vector } & $S$ & Number of susceptible vectors \\
\hline & $L$ & Number of incubating (latent) vectors \\
\hline & $V$ & Number of infected vectors \\
\hline & $N$ & Total number of vectors $(=S+L+V)$ \\
\hline \multicolumn{3}{|l|}{ Parameters } \\
\hline \multirow[t]{10}{*}{ Vector } & $\rho$ & Daily recruitment rate $\left(=\rho_{0}-\rho_{s} N\right.$ in logistic case, $\mu(1+\delta \cos (\theta t)$ in seasonal case $)$ \\
\hline & $\rho_{0}$ & Basic recruitment rate in logistic case \\
\hline & $\rho_{s}$ & Strength of density dependence in recruitment in logistic case \\
\hline & $\delta$ & Degree of seasonality in $\rho$ \\
\hline & $\theta$ & Regulates length of cycle in seasonal recruitment \\
\hline & $T$ & Period of seasonal function \\
\hline & $\alpha$ & Interval between bloodmeals on AHS hosts \\
\hline & $\beta$ & Proportion of vectors which become infected after biting an infective host \\
\hline & $\mu$ & Daily mortality rate $($ lifespan $=1 / \mu)$ \\
\hline & $\gamma$ & Daily virus development rate (duration of latent period $=1 / \gamma$ ) \\
\hline \multirow[t]{5}{*}{ Hosts } & $a_{i}$ & Biting rate on hosts of species $i$ \\
\hline & $b$ & Proportion of hosts which become infected following the bite of an infective midge \\
\hline & $r_{i}$ & Recovery rate for species $i$ (duration of infection $1 / r_{i}$ ) \\
\hline & $c_{i}$ & Mortality rate of infected hosts of species $i$ \\
\hline & $q$ & Proportion of hosts which are of species $1, H_{1} /\left(H_{1}+H_{2}\right)$ \\
\hline
\end{tabular}

Infective hosts, $y$. Hosts become infected as above. Hosts die of the disease at a rate $c$ and recover from the disease at a rate $r$ (the average duration of infectiveness in a surviving host is $1 / r$ ). This gives

$$
\frac{d y}{d t}=a b\left(\frac{N}{H}\right) x v-r y-c y .
$$

Immune hosts, $z$. Hosts become immune by recovering from the infection as above. Immunity is lifelong, so hosts do not leave this compartment and

$$
\frac{d z}{d t}=r y
$$

Total host population, $H$. As hosts are not replaced during an epidemic, we note that the total host population declines during an epidemic at a rate $c$, so

$$
\frac{d H}{d t}=-c Y
$$

Susceptible vectors, $S$. We are not explicitly considering immature stages, so the recruitment rate, $\rho$, is the rate of newly emerged female midges entering the adult population. Since we are assuming that the midge population remains constant, we do not need to include a time lag in recruitment. We assume transovarial transmission does not occur in Culicoides (Jones \& Foster, 1971; Allingham \& Standfast, 1990), although it may be important for other vectors such as mosquitoes and phlebotomines (e.g. Turell, 1988; Mitchell, 1991; Tesh et al., 1992). Thus, all newly emerged females enter the susceptible class. Recruitment into the susceptible population therefore is $\rho N$, where $N=S+L+$ $V(L$ and $V$ are the number of vectors in the latent and infective classes, respectively). This also assumes that there is no impact of the virus on reproduction and that all individual vectors are equally susceptible to infection. All classes of vectors have the same mortality rate, $\mu$ (the average lifespan of adult vectors is $1 / \mu$ ); there is no infection-induced mortality. For our assumption of a constant population size, necessarily $\rho=\mu$. Transmission from hosts to vectors depends on the biting rate, $a$; the proportion of hosts which are infected, $y$; the number of vectors that are susceptible, $S$; and the proportion of bites on an infected host which infect the vector, $\beta$ (note that any heterogeneity in susceptibility in the vector population is implicitly included in $\beta$ ). The transmission term then is $a \beta S y$ and

$$
\frac{d S}{d t}=\rho(S+L+V)-a S y-\mu S .
$$

Latent vectors, $L$. Vectors are infected as above. They leave the class by dying $(\mu)$ or by becoming infective at a rate $\gamma$ (the average duration of the latent period in surviving adult vectors is $1 / \gamma)$. The duration of the latent period can be modelled either with a constant rate or a fixed delay. Here, we use a constant rate for mathematical simplicity in later expansions. This gives

$$
\frac{d L}{d t}=a \beta S y-\mu L-\gamma L
$$



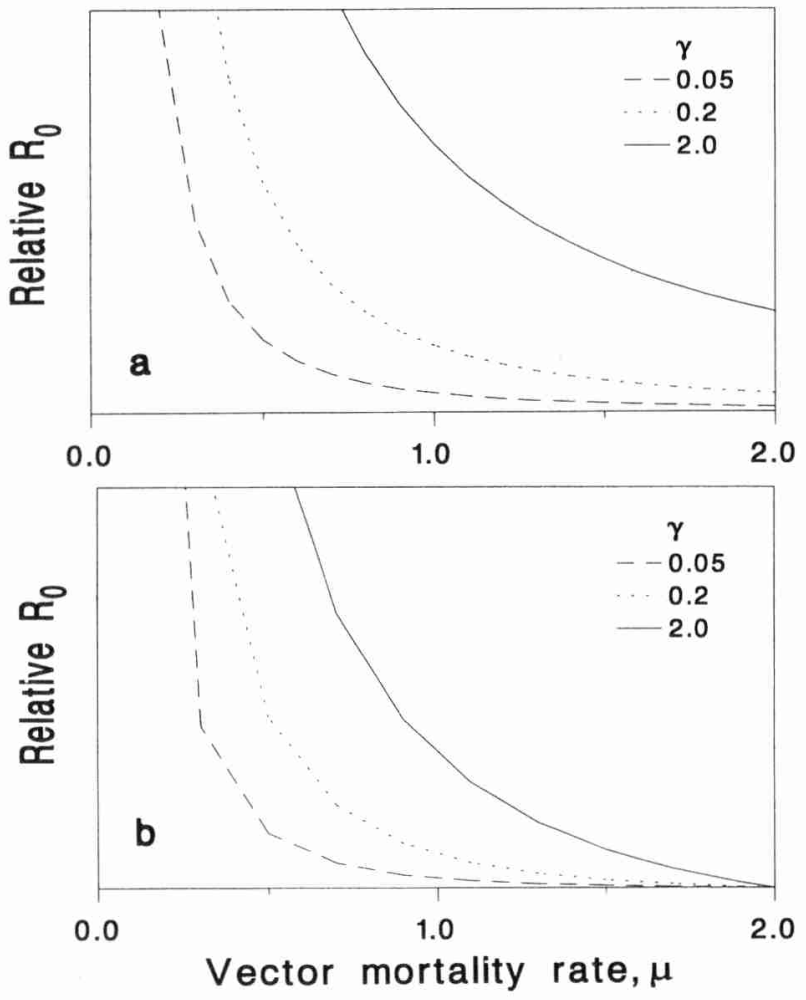

Fig. 2. The relationship between $R_{0}, \gamma$ and $\mu$. (a) The basic model; $N=1990$. (b) Explicit density-dependence in vector population dynamics. In this and following graphs, the $y$-axis is unscaled as only the particular parameters shown are included. In this case, the axes are scaled identically for comparison; $N$ in (a) is equal to $N$ in (b) when $\mu=0.01$.

Infective vectors, $V$. Vectors enter the infective class after the latent period, as above. The mortality rate is again $\mu$. As there is no recovery,

$$
\frac{d V}{d t}=\gamma L-\mu V
$$

For this basic model, $R_{0}$ is easily derived. The mathematical theory is given in the appendix. For an heuristic derivation (Anderson \& May, 1991), consider one primary case in a host. The duration of infectiveness is $1 /(r+c)$, during which time there is an average of $a(N / H)$ bites by susceptible midges. Of these, $\beta$ will result in an infected midge, so we have $a(N / H) \beta(r+c)$ infected midges. A fraction $\gamma(\gamma+\mu)$ of these will survive the latent period to become infective. These midges will survive, on average, for $1 / \mu$ days and bite at a rate $a$ during this time. Finally, $b$ of these bites will lead to infection of susceptible hosts. Thus,

$$
R_{0}=\frac{a^{2} b \beta}{(r+c) \mu} \frac{N}{H}\left(\frac{\gamma}{\gamma+\mu}\right)
$$

From this equation, it can be seen that the biting rate, $a$, is especially important in determining $R_{0}$, as it occurs squared, as has been discussed many times before (Ross, 1911; Macdonald, 1957; Aron \& May, 1982; Anderson \& May, 1991). Note, however, that this is the biting rate only on those hosts which are susceptible to the disease agent (in this case, the AHS virus). Other sources of bloodmeals reduce $a$, and thus $R_{0}$. The biting rate on AHS hosts depends on a combination of factors, such as the total biting rate (the inverse of the interval between bloodmeals from all hosts), relative host abundances and host preferences. In many cases it may be easier to measure these parameters and then calculate the biting rate on AHS hosts.

$R_{0}$ has a linear relationship with $b$ and $\beta$, and is inversely related to the rate at which individuals leave the infective class. $(r+c)$. There is also a linear relationship between $R_{0}$ and $(N / H)$, however, the relationship between $R_{0}$ and either component will depend on the relationship between $N$ and $H$. This will be discussed in more detail in the following section.

$R_{0}$ is also dependent on the midge mortality rate, $\mu$, and the virus development rate, $\gamma$ (Fig. 2a). Clearly $R_{0}$ is more sensitive to changes in both parameters when they are small.

\section{Population dynamics of midges}

We have been considering the case where the midge population is constant. This requires implicit and extreme density dependence, with $\rho$ necessarily equal to $\mu$. This is unrealistic, and we would like to explore the effect on $R_{0}$ of using a more realistic population growth function for $N$.

The familiar logistic equation is derived from birth and death rates which are linear functions of $N$ (Wilson \& Bossert, 1971), decreasing and increasing respectively with increasing $N$. Density has been shown to affect larval development and survival in mosquitoes (Service, 1993) and midges (although only in laboratory studies; Akey et al., 1978). We restrict our attention to the case of varying $\rho$ and constant $\mu$, with $\rho$ a linear function of $N$ (the dynamics of $N$ are very similar when both parameters are allowed to vary). This leads to:

$$
\frac{d S}{d t}=\left(\rho_{0}-\rho_{S} N\right) N-a \beta S y-\mu S
$$

for susceptible midges. All other equations will be the same as equations 2-7. $\rho_{s}$ and $\rho_{0}$ are the strength of the density-dependence in recruitment and the recruitment rate in the absence of density dependent constraints, respectively.

This regulates the midge population to a constant value, $N^{*}=\left(\rho_{0}-\mu\right) / \rho_{S^{*}}$ In this case the midge population will return to $N^{*}$ following a disturbance and $\rho$ is no longer constrained to be equal to $\mu$. The same equation (8) will apply for $R_{0}$. Substituting in the above expression for $\mathrm{N}^{*}$,

$$
R_{0}=\frac{a^{2} b \beta}{(r+c)}\left(\frac{\gamma}{\gamma+\mu}\right)\left(\frac{\rho_{0}-\mu}{\rho_{\mathrm{s}} \mu H}\right)
$$

As a consequence of the dependence of $R_{0}$ on $N, R_{0}$ is inversely related to $\rho_{S}$, decreasing as the strength of the density-dependence increases, and is linear with $\rho_{0}$. More important is the effect the explicit density dependence has on the relationship between $R_{0}, \mu$ and $\gamma$ (Fig. 2b), increasing the sensitivity of $R_{0}$ to $\mu$ when $\mu$ is small.

In the basic model we assumed that there was no relationship between $N$ and $H$. In this case, increasing $H$ will decrease $R_{0}$, as the bites by vectors on infected hosts are diluted by the increased 


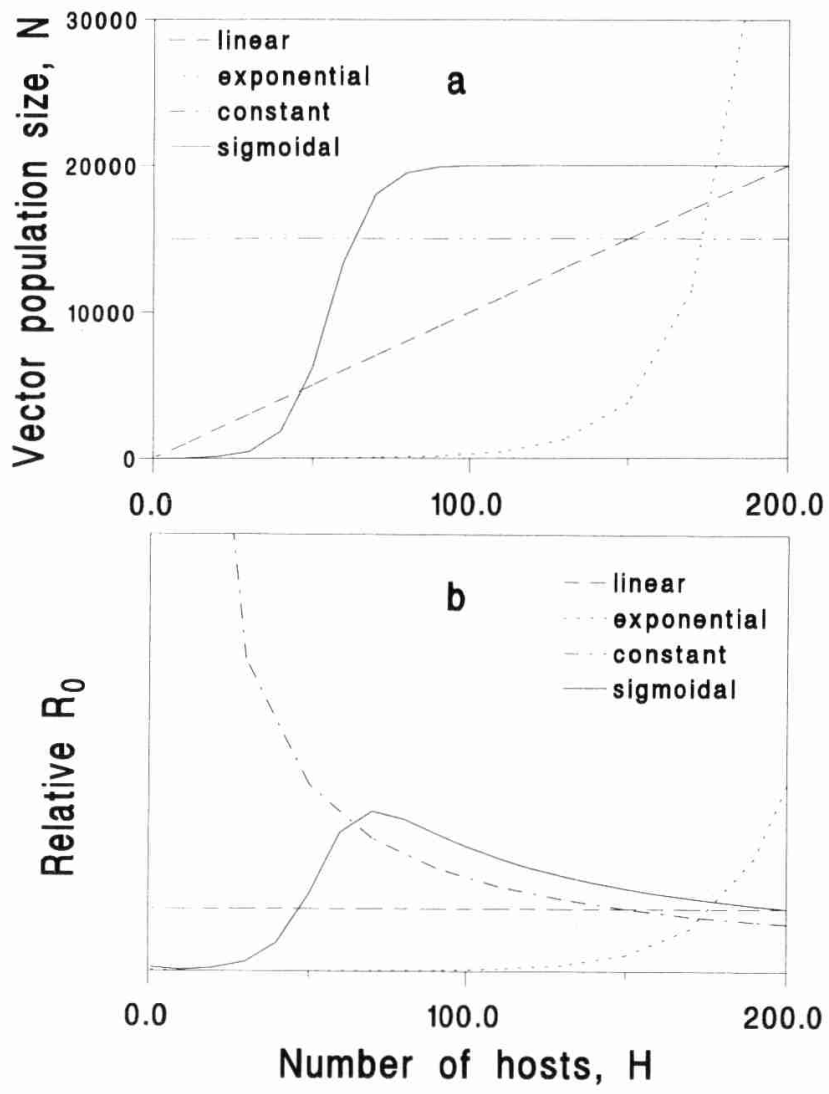

Fig. 3. Relationship between $N, H$ and $R_{0}$. (a) Four possible forms for the relationship between $N$ and $H$. (b) The effect of each of these relationships on $R_{0}$. Linear, $N=100(H)$; exponential, $N=e^{0.055 H}$; sigmoidal, $N=20000 /\left[1+4000 e^{-0.15 H}\right]$; constant (i.e. basic model), $N=15000$.

number of uninfected hosts. This may occur when other animals are the major source of bloodmeals for the vectors, but if the disease hosts are also the primary bloodmeal source $N$ may depend on $H$. It is likely that midge populations will increase as the host population increases, but the specific relationship may vary. Four examples of possible relationships between $N$ and $H$, and the effect on $R_{0}$, are shown in Fig. 3. A linear relationship between $N$ and $H$ results in $R_{0}$ being independent of $H$, whereas an exponential function leads to a steeply increasing relationship between $R_{0}$ and $H$. Interestingly, if the relationship is sigmoidal (where there is a maximum $N$ ), the relationship between $R_{0}$ and $H$ is humped Initially, $R_{0}$ increases as $N$ increases. Once $N$ reaches its maximum, however, $R_{0}$ decreases with additional hosts as the vector to host ratio decreases. In this case the effect of changing the host population on $R_{0}$ will depend on the original population size.

\section{Seasonality}

Midges may be highly seasonal in their population dynamics (Walker, 1977; Braverman et al., 1985; Mohammed \& Mellor, 1990; Capela et al., 1993; Greiner et al., 1993), as may other vectors such as mosquitoes (Reeves et al., 1994). There can be many factors involved in this seasonality, such as temperature, rainfall or photoperiod. Here we will consider only temperaturedependent parameters and their impact on $R_{0}$.

Seasonal peaks in abundance may depend on temperature through several different factors, either alone or in combination. Larval development of most Diptera is temperature dependent (Akey et al., 1978; Vaughan \& Turner, 1987; Allingham, 1991; Clements, 1992; Service, 1993; Tesh et al., 1992; Narladkar et al., 1993), which would lead to a temperature dependence in recruitment to the adult population. Similarly, temperature dependence in bloodmeal digestion or egg development (Mullens \& Holbrook, 1991; Clements, 1992) will result in temperature dependence in adult recruitment. Mortality rates may also be temperature dependent (Hunt et al., 1989; Reeves et al., 1994), which will affect the seasonality of vector abundance. However, generally recruitment will be much more variable (Akey et al., 1978; Hunt et al., 1989).

We wish to incorporate the essential features of a seasonally varying vector population, while keeping our model as mathematically tractable as possible. We therefore use a sinusoid function for $\rho$, but keep $\mu$ constant (the net effect on the dynamics of $N$ is very similar with $\mu$ seasonal as with $\mu$ constant). We restrict our attention to vector populations in a demographic steady-state, e.g. not increasing or decreasing on average, although varying seasonally (Fig. 4). This requires that the average recruitment rate be equal to the mortality rate. This leads to $\rho=\mu(1+\delta \cos (\theta t))$, where $\delta$ is the amplitude of the seasonal fluctuation in $\rho$ ( $\theta$ is a scaling factor for cycle length). The result is a vector population that varies in time like a sinusoidal curve for small $\delta$. This is similar to the model used by Aron \& May (1982), where the emergence of adult mosquitoes was assumed to vary sinusoidally.

The seasonal fluctuation in $\rho$ can be easily related to fluctuations in temperature. The yearly fluctuations in long-term (e.g. 40 years) average monthly temperatures can often be approximated quite well with sinusoid curves. If we then assume recruitment to be a linear function of temperature, we arrive at the sinusoid function for recruitment used above.

$R_{0}$ cannot be derived directly in this situation. Heesterbeek $\&$ Roberts $(1994 b, 1995)$ have developed other estimators of thresholds for the introduction of parasites into systems with a

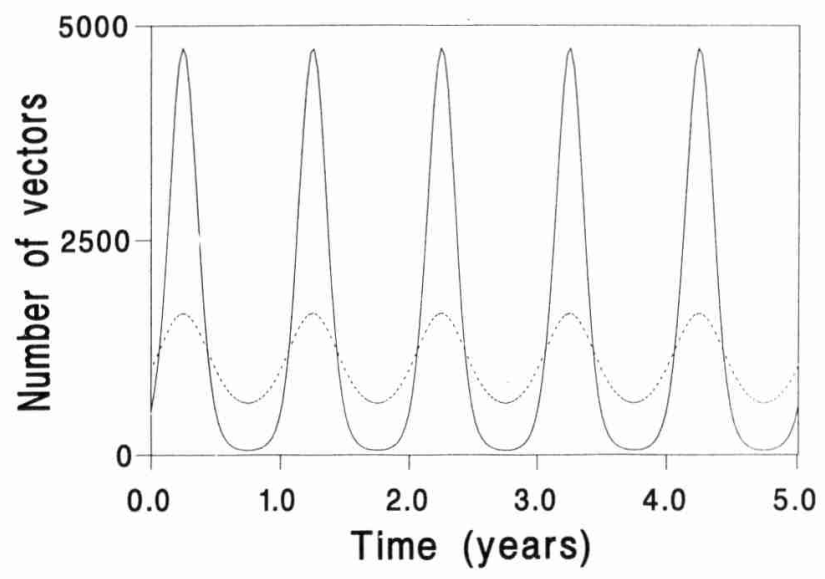

Fig. 4. Steady-state seasonal midge populations. $N(t)=N(0) \mathcal{E}^{\mu \delta \text { sin }(\theta t)}$. Solid line, $\delta=9, N(0)=500$; dotted line, $\delta=2, N(0)=1000 . \mu=0.25, \theta=$ 0.0172 for both. 
seasonal driving function. For our one-host model with seasonal recruitment, one of these estimators is

$$
\begin{aligned}
P & \approx\left(\frac{\gamma}{\gamma+\mu}\right)\left(\frac{a^{2} b \beta N(0)}{\mu H(r+c)}\right)[1+\mu \delta \psi] \\
& \approx R_{0}[1+\mu \delta \psi] .
\end{aligned}
$$

The derivation of this expression is in the appendix. When $\delta$ is 0 , this equation collapses to equation $8 . N(0)$ is the size of the vector population when the infection is introduced. $\psi$ depends on the relative timing between the peaks in prevalence in the hosts (e.g. the time of peak $y$ ) and of the vector population, and so can be either positive or negative. If prevalence in hosts is exactly in-phase with the vector population, $\psi$ will have its maximum possible value and the estimator will be increased from the non-seasonal $R_{0}$. If, however, the two peaks are exactly outof-phase, $\psi$ will have its minimum possible (negative) value and $P$ will be smaller than the non-seasonal $R_{0}$.

\section{Two-host types}

Many vector-borne diseases do not affect only one host. In some cases we are concerned with one host (usually either humans or domestic animals), and the other host types serve as a reservoir or as a bloodmeal source for vectors. In other cases more than one host type is of veterinary or medical interest. AHS infects most types of equines (Binepal et al., 1992), and there are known differences in disease-induced mortality rates and suspected differences in the duration of infectiveness. Horses, usually the host of primary concern, have a much higher mortality rate and a shorter duration of infection than other types of equids (Mellor, 1993; Rodriguez et al., 1992, 1993). Therefore the design of adequate control programmes will depend upon the importance of the different host types. Previous two-host models (Rogers, 1988; Sota \& Mogi, 1989; Hasibeder et al., 1992) have been concerned primarily with deriving $R_{0}$ based on systems at equilibrium, whereas we are more specifically interested in the invasion of the virus into a naive population.

Adding the second host type is a straightforward expansion of the model. We assume that the basic biology of transmission and disease is the same for all host types, and only the parameter values differ. Infections of midges from each host type are summed to give the total infection rate. For the hosts, we introduce typespecific parameters for $a, r$ and $c$ (subscripted to indicate host type) to equations $1-3$. Potentially, both $b$ and $\beta$ would also vary between host types, but there is very little information available. For the vectors, we replace $a \beta S y$ with $\left(a_{1} y_{1}+a_{2} y_{2}\right) \beta S$ in equations 5 and 6 , to include transmission from both host types. The resulting model is similar to previous two-host models, modified to reflect the specifics of the AHS system and using rate parameters rather than fixed time lags to describe the latent period.

The biting rates, $a_{1}$ and $a_{2}$, depend on the relative abundance of the two host types and host preferences of the vector. If we assume that there is no preference, the biting rates are determined by the proportion of each type of host and the total biting rate on both hosts, $a\left(=a_{1}+a_{2}\right)$. If we define $q$ as the proportion of hosts that are of type $1, H_{1} /\left(H_{1}+H_{2}\right)$, then $a_{1}=q a$ and $a_{2}=(1-q) a$.
The ratio of bloodmeals on host 1 to those on host 2 is then $q /(1-q)$, or the ratio of the two populations $\left(\mathrm{H}_{1} / \mathrm{H}_{2}\right)$.

Again, the formal derivation of $R_{0}$ is left for the appendix. Intuitively, it is the sum of the contribution to $R_{0}$ by each host type. Substituting for $a_{1}$ and $a_{2}$, in equation A.16 and rearranging to group parameters that are independent of host type, we obtain

$$
R_{0}=\frac{N \beta b a^{2}}{\mu}\left(\frac{1}{\left(H_{1}+H_{2}\right)}\right)\left(\frac{\gamma}{\gamma+\mu}\right)\left[\frac{q}{\left(r_{1}+c_{1}\right)}+\frac{(1-q)}{\left(r_{2}+c_{2}\right)}\right] .
$$

To explore the impact of two host types coexisting, consider four different host types, with varying recovery and mortality rates: high mortality and short infective period $(r+c=0.25)$; lower mortality and a longer infective period $(r+c=0.06)$; negligible mortality and very long infective period $(r+c=0.03)$; and a host which is marginally competent for the virus but serves as a bloodmeal host for the vector $(r+c=10)$.

The relative sizes of the two host populations affect $R_{0}$ (Fig. $5 \mathrm{a})$, depending on how different the net rate of loss of infectives $\left(r_{\mathrm{i}}+c_{\mathrm{i}}\right)$ is between the two hosts. Note that for $R_{0}$, it does not matter if hosts are removed by mortality or recovery, although this may affect control strategies. Replacing hosts of one type with another (moving along the $q$ axis in Fig. 5a) can either increase or decrease $R_{0}$. If any of the other transmission parameters vary with host type (e.g. $b$ or $\beta$ ), these will also affect the relative contribution of the two host types to $R_{0}$ (as given by equation 14 , with appropriate subscripting). As susceptibility to infection is included in $b$, this might well vary between host types in many systems.
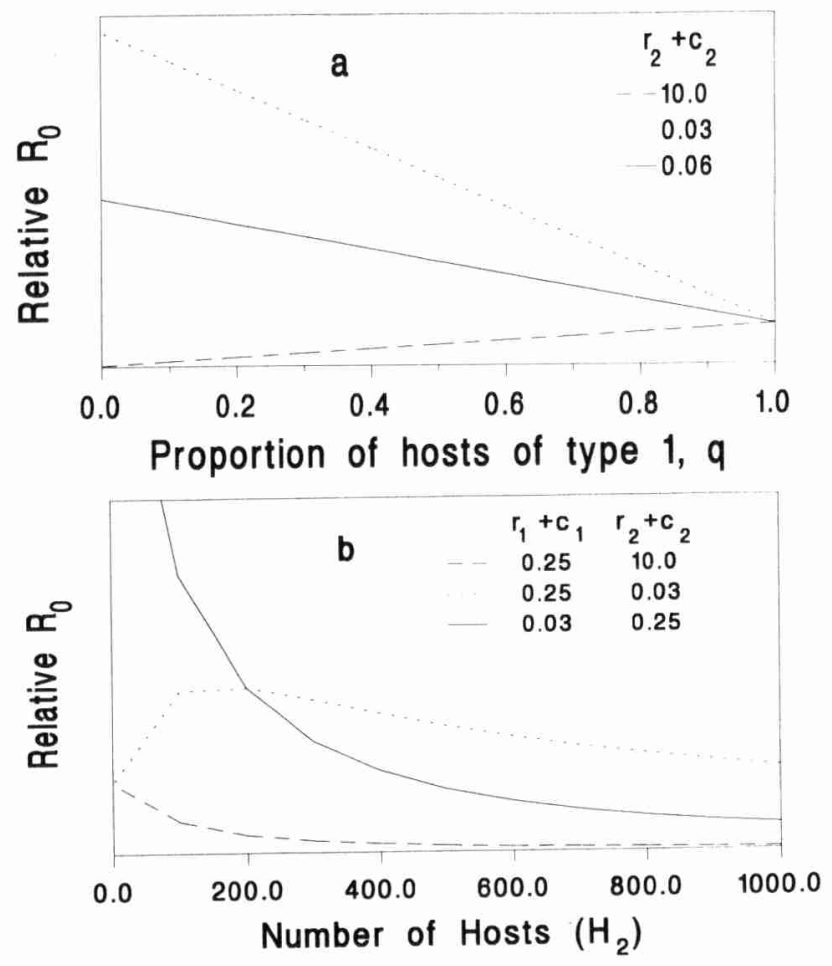

Fig. 5. Two host types. (a) The effect of varying q, the proportion of hosts of type 1. The total number of hosts is constant; $r_{1}+c_{1}=0.25$. (b) Addition of hosts of type 2 with varying values of $r_{2}+c_{2} ; H_{1}=200$. 
Considering equation 13 and the definition of $q$, it is clear that the overall host population size is important, as well as the relative proportions. The effect of adding hosts of one type will again depend on the relative removal rates. If $r_{1}+c_{1}>2\left(r_{2}+c_{2}\right)$, the relationship between $R_{0}$ and $H_{2}$ will be humped. Initially, $R_{0}$ increases as more of the highly competent host is added. At the point where $H_{2}=H_{1}\left[1-2\left(r_{2}+c_{2}\right) /\left(r_{1}+c_{1}\right)\right]$, this increase is offset by the decreasing vector to host ratio and $R_{0}$ declines (e.g. $r_{2}+c_{2}=0.03$ in Fig. 5b). Otherwise, if the number of the less competent host is increased (while vector numbers remain constant), the bites on the more competent host are diluted and $R_{0}$ declines.

\section{Discussion}

Various formulations of $R_{0}$ have been used in designing control programmes for vector-borne disease in the past, but few of these have included density-dependent or seasonal vector dynamics or multiple host types. This analysis shows that these factors need to be considered.

With resources often limited, it is important to be able to focus field and laboratory work on those aspects of a disease system with the highest potential for reducing disease. By deriving $R_{0}$ for various cases, we can identify those factors which will have the most effect on the ability of a disease agent to invade. Of particular interest are those parameters which have non-linear effects on $R_{0}$, as changing these will have the greatest impact. Interactions between parameters may also be important. The sensitivity of $R_{0}$ to each of the parameters of the basic model has been discussed exhaustively (Anderson \& May, 1991, and references therein), and we concentrate on the extensions to the model.

Less severe density-dependence in the vector population dynamics changed the sensitivity of $R_{0}$ to the vector mortality $(\mu)$ and the virus development rate $(\gamma)$, increasing the sensitivity of $R_{0}$ to $\mu$. Many insect vectors have adult lifespans in the range of $2-10$ days $(\mu=0.1-0.5)$, a range where $R_{0}$ is sensitive to both $\mu$ and $\gamma$. Therefore good estimates of both of these parameters will be necessary in determining the risk of virus invasion.

The functional relationship between vector and host abundance affects the impact of host abundance on $R_{0}$. Increasing host abundance (e.g. by introducing new stock) may decrease $R_{0}$ (as predicted by the basic model) or may increase it, and this may depend on the initial host abundance. In order to predict the relative risks of epidemics at different host abundances it is necessary to study the relationship between vector abundance and host abundance, and the mechanisms underlying this relationship.

The effect of a seasonal environment on the estimate of $R_{0}$ is complex. The primary effect is likely to be due to fluctuations in vector abundance, although other factors may also be important. For example, virus development rates vary with temperature in some systems (Lundström et al., 1990; Turell \& Lundström, 1990; Turell, 1993; P.S.M., unpublished data), as may mortality rates (see references given earlier). This may have epidemiological consequences if the fraction surviving the latent period fluctuated significantly.

Previous discussions have suggested (Anderson, 1993) that $R_{0}$ may fluctuate in synchrony with vector population abundance. We have shown here that this effect will be moderated by a parameter representing the phasing of fluctuations in vector abundance with fluctuations in the infective host population. This parameter will be greatest when seasonal fluctuations are large and when infection is introduced such that the peak numbers of infective hosts occur at the time of peak vector abundance. Simulation studies of a similar AHS model suggest (C.C.L. and M.E.J.W., unpublished data) that this phasing is dependent on the time of year when the virus is introduced, and that introductions during the increasing phase of vector abundance are most likely to result in an epidemic. This effect has not previously been described and its importance in practice is unknown. Long latent or infective periods in the host may affect this phasing, and hence the impact of seasonality. Data on fluctuations in both numbers of infected hosts and number of vectors during an epidemic are needed to explore this aspect further.

The expansion of the model to two host species indicated that it is the difference in their contribution to $R_{0}$ that is important. A similar effect would be seen in extensions to more than two host species. For the AHS example, we have illustrated this using differences in the relative rate of loss of infectives, although there may also be differences in other parameters (such as the transmission rates) which are also important. A comparative approach has been adopted previously for the parameters of vector competency (Dye, 1992; Hasibeder et al., 1992), and is clearly relevant to multiple host types as well. This analysis is useful in exploring the potential contribution of reservoir hosts to $R_{0}$. The presence of a less competent host can lower $R_{0}$ by lowering the vector-host ratio, although this is sensitive to any functional relationships between vector and host abundances (as discussed above). The presence of a more competent host may increase $R_{0}$, but only over a limited range of reservoir host abundance, beyond which increasing reservoir host abundance may decrease $R_{0}$ (with the same caveats as before). The impact of control strategies which rely on manipulating the numbers of two or more hosts also depends on their relative competence. There are conditions where the removal of a reservoir host could increase $R_{0}$, and hence the risk of epidemics in the hosts it is intended to protect.

In addition to indicating which parameters have the most impact, formulating the model can indicate other areas for research. These models make many assumptions which need to be tested. The relationship between the number of hosts and the number of vectors should be elaborated further; possibly recruitment to the adult vector population should be a function of host availability as well as temperature or vector population size. The choice of linear functions for the temperature relationships were made in the absence of data and for mathematical convenience; these also need to be tested empirically.

For AHS the primary concern is the prevention of epidemics among horses. This may be achieved by vaccination (Rodriguez et al., 1993), with the fraction which must be effectively vaccinated being higher when $R_{0}$ is large (Anderson \& May, 1991). As we have shown, this may depend in complex ways on the abundance of both horses and of reservoir hosts and their relative contributions to transmission. A second option is vector control (Rodriguez et al., 1993), with the necessary reduction in midge numbers and/or life-expectancy also being greater when $R_{0}$ is large. We have shown that this may depend on the severity 
of density-dependence and the degree of seasonality in midge population dynamics. More information on these components is needed both to understand fully the epidemiology of AHS and to make informed decisions regarding the design of control programmes.

\section{Acknowledgments}

Support for this research was provided by a BBSRC Link Grant and CEC (contract nos. 8001-CT91-0211 and TS3-CT92-0151). M.E.J.W. is supported by the Royal Society. J.A.P.H. gratefully thanks the Department of Zoology (Oxford) for its hospitality during the start of this research and the Netherlands Organization for Scientific Research (NWO) and the Royal Society for financial support. We thank Peter Rawlings, Dan Haydon and Matthew Baylis for discussions and review of the manuscript.

\section{Appendix}

\section{One host}

The mathematical theory needed to define and calculate $R_{0}$ in heterogenous populations can be found in Diekmann et al. (1990; earlier references are summarized in Anderson \& May, 1991). For the special case where only a finite number of different individuals are recognized, a more accessible presentation of the same theory is given in Heesterbeek \& Roberts (1994a). The problem that arises when trying to define and calculate $R_{0}$ in heterogenous populations can be easily illustrated for the case where only two types of individuals are recognized. This is the situation in the single-host model in section A where we have to deal with hosts and vectors. One can try to calculate in principle four 'reproduction numbers' based on the definition of $R_{0}$ in a homogenous population: the expected number of new cases caused by one infected individual. In our situation we could easily write down expressions for 1 , the expected number of midges infected by one infected host, and 2, the expected number of hosts infected by one infected midge. Since hosts do not infect other hosts directly (and the same for midges) the other two possible 'reproduction numbers' are zero. We then have four numbers and the question is how these should be averaged in order to obtain something that has the threshold property of leading to a successful invasion if it is bigger than one, a failed invasion if it is less than 1 , and retains the interpretation of the homogenous $R_{0}$.

Let the index ' 1 ' refer to hosts, and ' 2 ' to midges, and write $k_{\text {i }}$ for the expected number of new cases of type $i$ individuals, caused by one infected individual of type $j$ during its entire period of infectivity, in a population consisting of susceptibles (i.e. we attach a symbol to each of the four 'reproduction numbers' introduced above). Note that $k_{11}=k_{22}=0$ in our case. We combine the four numbers in a matrix

$$
K=\left(\begin{array}{ll}
0 & k_{12} \\
k_{21} & 0
\end{array}\right)
$$

From the interpretations of the parameters it is clear that

$$
\begin{aligned}
& k_{12}=\frac{a b \gamma}{\mu(\gamma+\mu)} \\
& k_{21}=a \frac{N}{H} \frac{\beta}{r+c}
\end{aligned}
$$

In general, the entries of $K$ are obtained by considering submodels for the transmission process that take the relevant idiosyncrasies of the particular system into account (see for example Diekmann et al., 1990). In our case, however, we can easily obtain the entries $k_{\mathrm{ij}}$ directly from the definition.

We assume that the susceptible populations are all in a demographic steady state prior to invasion and that the decrease in the susceptible population sizes due to infection can be neglected in the initial phase of the invasion. These are reasonable assumptions given that we look only at the beginning of a possible epidemic, i.e. a small number of infectives will bring the infection into a large population of susceptibles, and the time-scale on which invasion success is decided is much shorter than the timescale of demographic change. In our case the host population is taken to be constant from the start (no demographics for the host), and the demographic steady state of the vector population prior to invasion is given by $N$.

Linear algebra can be used to show that the dominant eigenvalue 1 of the matrix $K$, i.e. the eigenvalue that is larger in absolute value than all other eigenvalues of $K$, has precisely the properties we are looking for and can therefore be taken as the correct way of averaging the four 'reproduction numbers' (e.g. see Caswell, 1989). Since for any number $\lambda>1$ if and only if $\lambda^{2}>1$, we can define

$$
R_{0}=\lambda^{2}=(\text { the dominant eigenvalue of } K) \text { squared, }
$$

where the exponent 2 reflects that it is a biological necessity in the host-vector case for the parasite to pass through two types of individuals in order to complete its life-cycle. The eigenvalues of $K$ are the solutions of $\lambda^{2-} k_{12} k_{21}=0$ and we therefore find

$$
R_{0}=k_{12} k_{21}=\frac{a^{2} b \beta}{(r+c) \mu}\left(\frac{N}{H}\right)\left(\frac{\gamma}{\gamma+\mu}\right)
$$

Note that in this case, as $k_{12} \geq 0$ and $k_{21} \geq 0$, necessarily $\lambda \geq 0$ (and hence $R_{0} \geq 0$ ).

\section{Seasonality}

The procedure to define and calculate $R_{0}$ as described above can no longer be used if the assumption that the susceptible populations are constant during the phase where success or failure of an invasion is decided no longer holds. However, if one or more of the susceptible populations are periodic at the moment of invasion, then something can be said about threshold quantities deciding the invasion question. This was developed in Heesterbeek \& Roberts (1995) for helminth infections and for micro-parasites in Heesterbeek \& Roberts (1994b). We will discuss only one of the threshold quantities defined there.

Prior to invasion, in the absence of infectives, the susceptible population of midges is described by equation (5) in the text, with $y=0$ and $r=\mu(1+\delta \cos (\theta t))$. This equation can be solved 
to give the periodically varying population of susceptible midges prior to invasion

$$
N(t)=N(0) e^{\mu \delta \sin (\theta t)} \approx N(0)+N(0) \mu \delta \sin (\theta t) \text { for } \mu \delta \text { small, (A.5) }
$$

i.e. the vector population varies sinusoidally for small $\mu \delta$. $N(0)$ is the vector population at $t=0$. The period of the recruitment function is $T$, with $T=2 \pi / \theta$. In our one-host, one-vector case one can define a quantity

$$
P=\frac{\int_{0}^{T} f_{2}(t) m_{21}(t) y(t) d t}{\int_{0}^{T} y(t) d t} \frac{\int_{0}^{T} f_{1}(t) m_{12}(t) V(t) d t}{\int_{0}^{T} V(t) d t}
$$

(see Heesterbeek \& Roberts, 1994b, 1995, for the general formulation), where the first quotient refers to infective midges being produced by infective hosts with $f_{2}(t)$ being the average duration of infectiousness in midges that become infected at time $t$ and $m_{21}(t)$ the average number of midges infected by one infective host at time $t$. Similarly, the second quotient refers to infected hosts produced by infective midges, with $f_{1}(t)$ the average duration of infectiousness in hosts that become infected at time $t$, and $m_{12}(t)$ the average number of hosts infected by one infective midge. One can show that $P$ thus defined has the threshold property we want, in that $P>1$ leads to a successful invasion into the periodic vector population, whereas for $P<1$ the infection cannot spread. We could allow any or all of the $m_{i j}$ 's or $f_{i}$ 's to depend periodically in time, but will first look just at periodic $N(t)$, so that $f_{1}=1 /(r+c), f_{2}=\gamma / \mu(\gamma+\mu), m_{12}=a b$ and the only time-dependent parameter, $m_{21}=a \beta N(t) / H$, where $N(t)$ is given by eqn A.5. In this case,

$$
P=\frac{a^{2} b \beta \gamma}{\mu(\gamma+\mu)(r+c) H} \frac{\int_{0}^{T} N(t) y(t) d t}{\int_{0}^{T} y(t) d t}
$$

If we approximate $N(t)$ as in eqn A.5, for $\mu \delta$ small we obtain

$$
P \approx R_{0}(1+\mu \delta \psi)
$$

where

$$
\psi=\frac{\int_{0}^{T} \sin (\theta t) y(t) d t}{\int_{0}^{T} y(t) d t}
$$

and where $R_{0}$ is given by eqn A.4. To evaluate the effects of the periodicity on $R_{0}$, we have to approximate the quotient $\psi$ or determine it numerically from the original system of differential equations. One could then draw graphs of $P$, the quantity that describes the ability of the parasite to invade, as a function of $\delta$, the parameter that describes the periodic behaviour of the vector population.

\section{Two hosts}

For the model in section $\mathrm{D}$, the case of two host-types and one vector-type, $K$ is a $3 \times 3$ matrix, where now the indices ' 1 ' and ' 2 ' refer to the two host species and ' 3 ' to the vector

$$
K=\left(\begin{array}{ccc}
0 & 0 & k_{13} \\
0 & 0 & k_{23} \\
k_{31} & k_{3} & 0
\end{array}\right)
$$

where

$k_{13}=\frac{a_{1} b \gamma}{\mu(\gamma+\mu)}$

$k_{23}=\frac{a_{2} b \gamma}{\mu(\gamma+\mu)}$

$k_{31}=a_{1}\left(\frac{N}{H_{1}}\right) \frac{\beta}{r_{1}+c_{1}}$

$k_{32}=a_{2}\left(\frac{N}{H_{2}}\right) \frac{\beta}{r_{2}+c_{2}}$.

Calculating $\lambda^{2}$ as before leads to

$R_{0}=k_{13} k_{31}+k_{23} k_{32}=\left(\frac{\gamma}{\gamma+\mu}\right)\left(\frac{a_{1}^{2} b \beta}{\left(r_{1}+c_{1}\right) \mu} \frac{N}{H}+\frac{a_{2}^{2} b \beta}{\left(r_{2}+c_{2}\right) \mu} \frac{N}{H_{2}}\right)$

Note that this is just the sum of the two reproduction numbers we would have attained had either host been considered alone, as one would intuitively expect.

\section{References}

Akey, D.H., Potter, H.W. \& Jones, R.H. (1978) Effects of rearing temperature and larval density on longevity, size, and fecundity of the biting gnat Culicoides variipennis. Annals of the Entomological Society of America, 71, 411-418.

Allingham, P.G. (1991) Effect of temperature on late immature stages of Culicoides brevitarsis (Diptera: Ceratopogonidae). Journal of Medical Entomology, 28, 878-881.

Allingham, P.G. \& Standfast, H.A. (1990) An investigation of transovarial transmission of Akabane virus in Culicoides brevitarsis. Australian Veterinary Journal, 67, 273-274.

Anderson, R.M. (1993) Epidemiology. Modern Parasitology (ed. by F. E. G. Cox), pp. 75-116. Blackwell Scientific Publications, Oxford.

Anderson, R.M. \& May, R.M. (1991) Infectious Diseases of Humans: Dynamics and Control. Oxford University Press.

Aron, J.L. \& May, R.M. (1982) The population dynamics of malaria. Population Dynamics of Infectious Disease (ed. by R. M. Anderson), pp. 139-179. Chapman \& Hall, London.

Binepal, V.S., Wariru, B.N., Davies, F.G., Soi, R. \& Olubayo, R. (1992) An attempt to define the host range for African horse sickness virus (Orbivirus, Reoviridae) in East Africa, by a serological survey in some Equidae, Camelidae, Loxodontidae and Carnivore. Veterinary Microbiology, 31, 19-23.

Braverman, Y., Linley, J.R., Marcus, R. \& Frish, K. (1985) Seasonal survival and expectation of infective life of Culicoides spp. (Diptera: Ceratopogonidae) in Israel, with implications for bluetongue virus transmission and a comparison of the parous rate in C.imicola from Israel and Zimbabwe. Journal of Medical Entomology, 22, 476-484.

Capela, R., Sousa, C., Pena, I. \& Caeiro, V. (1993) Preliminary note on the distribution and ecology of Culicoides imicola in Portugal. Medical and Veterinary Entomology, 7, 23-26.

Caswell, H. (1989) Matrix Population Methods. Sinauer, Sunderland, Mass.

Clements, A.N. (1992) The Biology of Mosquitoes, Vol. 1. Development, Nutrition and Reproduction. Chapman \& Hall, London.

Diekmann, O., Heesterbeek, J.A.P. \& Metz, J.A.J. (1990) On the definition and the computation of the basic reproductive rate $R_{0}$ in models for infectious disease in heterogeneous populations. Journal of Mathematical Biology, 28, 365-382. 
Dye, C. (1992) The analysis of parasite transmission by bloodsucking insects. Annual Review of Entomology, 37, 1-19.

Greiner, E.C., Mo, C.L., Homan, E.J., Gonzales, J., Oviedo, M.T., Thompson, L.H., Gibbs, E.P.J. \& the Regional Bluetongue Team. (1993) Epidemiology of bluetongue in Central America and the Caribbean: initial entomological findings. Medical and Veterinary Entomology, 7, 309-315.

Hasibeder, G., Dye, C. \& Carpenter, J. (1992) Mathematical modelling and theory for estimating the basic reproduction number of canine leishmaniasis. Parasitology, 105, 43-53.

Heesterbeek, J.A.P. \& Roberts, M.G. (1994a) Mathematical models for microparasites of wildlife. In: Ecology of Infectious Diseases in Natural Populations (ed. by B. T. Grenfell and A. P. Dobson). Cambridge University Press.

Heesterbeek, J.A.P. \& Roberts, M.G. (1994b) Threshold quantities for infectious diseases in periodic environments. Proceedings of the Second European Conference on Mathematics Applied to Biology and Medicine (ed. by P. Auger and J. Demongeot). Wuerz Publications.

Heesterbeek, J.A.P. \& Roberts, M.G. (1995) Threshold quantities for helminth infections. Journal of Mathematical Biology, in press.

Hunt, G.J., Tabachnick, W.J. \& McKinnon, C.N. (1989) Environmental factors affecting mortality of adult Culicoides variipennis (Diptera: Ceratopogonidae) in the laboratory. Journal of the American Mosquito Control Association, 5, 387-3291.

Jones, R.H. \& Foster, N.M. (1971) Transovarian transmission of bluetongue virus unlikely for Culicoides variipennis. Mosquito News, 31, 434437.

Lundström, J.O., Turell, M.J. \& Niklasson, B. (1990) Effect of environmental temperature on the vector competence of Culex pipiens and Cx torrentium for Ockelbo virus. American Journal of Tropical Medicine and Hygiene, 43, 534-542.

MacDonald, G. (1957) The Epidemiology and Control of Malaria. Oxford University Press.

Mellor, P.S. (1993) African horse sickness: transmission and epidemiology. Veterinary Research, 24, 199-212.

Mitchell, C.J. (1991) Vector competence of North and South American strains of Aedes albopictus for certain arboviruses: a review. Journal of the American Mosquito Control Association, 7, 446-451.

Mohammed, M.E.H. \& Mellor, P.S. (1990) Further studies on bluetongue and bluetongue-related orbiviruses in the Sudan. Epidemiology and Infection, 105, 619-632.

Mullens, B.A. \& Holbrook, F.R. (1991) Temperature effects on the gonotrophic cycle of Culicoides variipennis (Diptera: Ceratopogonidae). Journal of the American Mosquito Control Association, 7, 588-591.

Narladkar, B.W., Shastri, U.V. \& Shivpuje, P.R. (1993) Studies on bionomics of Culicoides spp. (Diptera: Ceratopogonidae) with a note on life cycle. Indian Veterinary Journal, 70, 313-316.

Reeves, W.C., Hardy, J.L., Reisen, W.K. \& Milby, M.M. (1994) Potential effect of global warming on mosquito-borne arboviruses. Journal of Medical Entomology, 31, 323-332.

Rodriguez, M., Hooghuis, H. \& Castaño, M. (1992) African horse sickness in Spain. Veterinary Microbiology, 33, 129-142.

Rodriguez, M., Hooghuis, H. \& Castaño, M. (1993) Current status of the diagnosis and control of African horse sickness. Veterinary Research, 24, 189-197.

Rogers, D.J. (1988) A general model for the African trypanosomes. Parasitology, 97, 193-212.

Ross, R. (1911) The Prevention of Malaria. Murray, London.

Scott, M.E. \& Smith, G. (ed) (1994) Parasitic and Infectious Diseases. Academic Press, New York.

Service, M.W. (1993) Mosquito Ecology: Field Sampling Methods. Elsevier, London.

Sota, T. \& Mogi, M. (1989) Effectiveness of zooprophylaxis in malaria control: a theoretical inquiry, with a model for mosquito populations with two bloodmeal hosts. Medical and Veterinary Entomology, 3, 337-345.

Tesh, R.B., Lubroth, J. \& Guzman, H. (1992) Simulation of arbovirus overwintering: survival of Toscana virus (Bunyaviridae: Phlebovirus) in its natural sand fly vector Phlebotomus perniciosus. American Journal of Tropical Medicine and Hygiene, 47, 574-581.

Turell, M.J. (1988) Horizontal and vertical transmission of viruses by insect and tick vectors. In: The Arboviruses: Epidemiology and Ecology, Vol. I (ed. by T. P. Monath). CRC Press, Boca Raton.

Turell, M.J. (1993) Effect of environmental temperature on the vector competence of Aedes taeniorhynchus for Rift Valley fever and Venezuelan equine encephalitis viruses. American Journal of Tropical Medicine and Hygiene, 49, 672-676.

Turell, M.J. \& Lundström, J.O. (1990) Effect of environmental temperature on the vector competence of Aedes aegypti and Ae.taeniorhynchus for Ockelbo virus. American Journal of Tropical Medicine and Hygiene, 43, 543-550.

Vaughan, J.A. \& Turner, E.C.J. (1987) Development of immature Culicoides variipennis (Diptera: Ceratopogonidae) from Saltville, Virginia, at constant laboratory temperatures. Journal of Medical Entomology, 24, 390-395.

Walker, A.R. (1977) Seasonal fluctuation of Culicoides species (Diptera: Ceratopogonidae) in Kenya. Bulletin of Entomological Research, 67, 217-233.

Wilson, E.O. \& Bossert, W.H. (1971) A Primer of Population Biology. Sinauer, Sunderland, Mass.

Accepted 6 March 1995 